\title{
Characterization of JG024, a pseudomonas aeruginosa PB1-like broad host range phage under simulated infection conditions
}

\author{
Julia Garbe ${ }^{1}$, Andrea Wesche¹, Boyke Bunk', Marlon Kazmierczak², Katherina Selezska ${ }^{3}$, Christine Rohde², \\ Johannes Sikorski ${ }^{2}$, Manfred Rohde ${ }^{3}$, Dieter Jahn¹, Max Schobert ${ }^{1 *}$
}

\begin{abstract}
Background: Pseudomonas aeruginosa causes lung infections in patients suffering from the genetic disorder Cystic Fibrosis (CF). Once a chronic lung infection is established, P. aeruginosa cannot be eradicated by antibiotic treatment. Phage therapy is an alternative to treat these chronic $P$. aeruginosa infections. However, little is known about the factors which influence phage infection of $P$. aeruginosa under infection conditions and suitable broad host range phages.

Results: We isolated and characterized a phage, named JG024, which infects a broad range of clinical and environmental $P$. aeruginosa strains. Sequencing of the phage genome revealed that the phage JG024 is highly related to the ubiquitous and conserved PB1-like phages. The receptor of phage JG024 was determined as lipopolysaccharide. We used an artificial sputum medium to study phage infection under conditions similar to a chronic lung infection. Alginate production was identified as a factor reducing phage infectivity.

Conclusions: Phage JG024 is a suitable broad host range phage which could be used in phage therapy. Phage infection experiments under simulated chronic lung infection conditions showed that alginate production reduces phage infection efficiency.
\end{abstract}

\section{Background}

Pseudomonas aeruginosa is well known as an opportunistic human pathogen characterized by a high intrinsic antibiotic tolerance [1,2]. In humans, $P$. aeruginosa can cause urinary tract, respiratory tract, and burn wound infections [3-5]. Respiratory tract infections caused by $P$. aeruginosa are dreaded in patients suffering from the genetic disorder Cystic Fibrosis (CF) [2,6,7]. CF patients exhibit an increased mucus production in the lung [8]. Bacteria like $P$. aeruginosa are able to colonize this mucus and cause chronic infections, which cannot be eradicated by antibiotic treatment [4]. Several hypothesis exist explaining the observed high antibiotic tolerance of $P$. aeruginosa in the CF-lung, which is caused by special growth conditions. These include growth as biofilm-like microcolonies, which have been shown to increase

\footnotetext{
* Correspondence: m.schobert@tu-bs.de

'Institute of Microbiology, Technische Universität Braunschweig,

Spielmannstr. 7, 38106 Braunschweig, Germany

Full list of author information is available at the end of the article
}

antibiotic tolerance up to 1000-fold [9,10]. A couple of in vitro model systems have been described to simulate a CF lung infection caused by $P$. aeruginosa [11-13]. The artificial sputum medium is a complex medium based on components measured in the CF sputum [12]. It mimics the CF-lung environment during infection and causes typical $P$. aeruginosa phenotypes as mucoidy and microcolony formation [12]. Since eradication of chronic $P$. aeruginosa infections by antibiotics fails, phage therapy is a possibility to treat bacterial infections. Advantages over antibiotics are the specificity of phages and that phages can be isolated and investigated rapidly [14]. For this reason, several suitable $P$. aeruginosa broad host range phages have been characterized. The Pseudomonas infecting PB1-like phages are widespread in nature and possess highly conserved genomes. Comparative genome analysis of five PB1-like (PB1, SN, 14-1, LMA2 and LBL3) phages was recently published and is the first genome report for these phages [15]. PB1-like phages belong to the Myoviridae phage family 
and the genome sizes vary between 64,427 and 66,530 bp. The genomes encode for 88 (LBL3) to 95 proteins (LMA2) [15]. More than 42 phages have been reported to be PB1-like. These results are mainly based on DNA hybridization and morphological studies $[15,16]$. More recently, PB1-like phages as phage 14-1 have been reported as part of a well defined phage cocktail to treat $P$. aeruginosa burn wound infections [17]. The application of phages as a therapeutical agent requires an in depth understanding of the phage biology [18]. Moreover, phages which multiply well under in vitro conditions can fail to replicate during treatment in vivo [19]. Therefore, phages and especially the ability of the phage to infect the host in vivo should be investigated carefully prior to use. Here we describe the in depth characterization of a broad host range PB1-like phage with a slight prevalence to clinical isolates. We used an artificial sputum medium to simulate the conditions in the CF lung and investigated the ability of phage JG024 to infect $P$. aeruginosa and multiply under these conditions.

\section{Results and Discussion}

\section{Isolation and host range of phage JG024}

Phages were isolated from sewage as described in Methods. We isolated $59 P$. aeruginosa specific phages and used an initial set of 5 different $P$. aeruginosa strains as the laboratory strains PAO1, PA14 as well as three clinical isolates (BT2, PACF15 and MH19, Table 1) to test the host range. One phage, which was named JG024, was able to conduct clear lysis on this set of bacterial strains. To determine the host range of JG024 in more detail, we used 19 clinical isolates from CF patients and from urinary tract infections as well as a collection of 100 environmental strains (Table 1). JG024 is able to infect $84 \%$ of all tested clinical isolates. Furthermore, JG024 is even capable of infecting a $P$. aeruginosa muc $A$ mutant and the clinical isolate BT73, which both showed the same mucoid phenotype. mucA mutants produce large amounts of the exopolysaccharide alginate and mutations in $m u c A$ are critical for the conversion of non-mucoid to mucoid $P$. aeruginosa variants in the lung of CF patients [20,21]. Additionally, we determined the host range of the phage JG024 with a collection of $100 P$. aeruginosa environmental strains isolated from different rivers (Oker, Aller, Weser) in Lower Saxony, Germany. The results showed that JG024 was able to infect $50 \%$ of the strains. Interestingly, phage JG024 showed a clear lysis for only $45 \%$ of the 50 lysed environmental isolates but was able to conduct clear lysis on $68 \%$ of the 19 lysed clinical isolates.

\section{Family affiliation of JG024}

To determine family affiliation of phage JG024, we determined the nature of the nucleic acids and the morphology of the phage to assign the family by comparison [22]. Nucleic acids were isolated as described in Methods and identified as dsDNA due to its sensitivity to restriction endonucleases like SacII, which cut only dsDNA. SacII produced distinct fragments of approximately $30 \mathrm{~kb}, 25 \mathrm{~kb}$ and $8 \mathrm{~kb}$ (data not shown). Computational analysis of the SacII restriction sites in the sequenced genome (see below) revealed slightly different

Table 1 Strains and phages used in this study.

\begin{tabular}{|c|c|c|}
\hline Bacterial strain or phage & Phenotype or genotype & Reference \\
\hline PAO1 & wild type & [48] \\
\hline PA14 & wild type & [49] \\
\hline FRD1 & mucoid CF isolate & [34] \\
\hline PAO1 $\triangle M U C A$ & PAO1 mucA::aacC1-gfp $\mathrm{Gm}^{\mathrm{R}}$ & $\begin{array}{c}\text { Sabrina Thoma, this laboratory, } \\
\text { unpublished }\end{array}$ \\
\hline PAO1 $\triangle$ pilA & $\begin{array}{c}\text { pilA inactivated by allelic displacement; tagged with } \\
\text { eGFP, TcR }{ }^{\mathrm{R}}, \mathrm{Gm}^{\mathrm{R}}\end{array}$ & {$[50]$} \\
\hline PAO1 $\triangle$ flim & $\begin{array}{l}\text { flim inactivated by allelic displacement; tagged with } \\
\qquad G G P, T c^{R}, G^{R}\end{array}$ & {$[50]$} \\
\hline PAO1 $\triangle a l g C$ & PAO1 algC :aacC1-gfp $\mathrm{Gm}^{\mathrm{R}}$ & $\begin{array}{l}\text { Julia Garbe, this laboratory, } \\
\text { unpublished }\end{array}$ \\
\hline BT2, BT72, BT73, RN3, RN43, RN45, NN84 & clinical CF isolates & $\begin{array}{l}\text { Medical Highschool Hannover, } \\
\text { Germany }\end{array}$ \\
\hline $\begin{array}{l}\text { PACF15, PACF21, PAKL1, PAKL4, PACF60, PACF61, } \\
\text { PACF62, PACF63 }\end{array}$ & clinical CF isolate & Gerd Döring, Tübingen, Germany \\
\hline Nr. $18,19,26,29$ & urinary tract infection isolate & Michael Hogardt, München, Germany \\
\hline Environmental strains & & $\begin{array}{c}\text { Katherina Selezska, HZI Braunschweig, } \\
\text { Germany }\end{array}$ \\
\hline $\mathrm{JG} 024$ & wild type PAO1 LPS specific lytic bacteriophage & this study \\
\hline
\end{tabular}


fragment sizes of $28,348 \mathrm{~kb}$ and $21,719 \mathrm{~kb}$, respectively as well as two fragments with a size of $8,49 \mathrm{~kb}$ and $7.718 \mathrm{~kb}$, which we observed as one $8 \mathrm{~kb}$ fragment.

Electron microscopy (Figure 1) shows an icosahedral head with a length of $80 \mathrm{~nm}$ and a width of $75 \mathrm{~nm}$. The contractile tail, which consists of a neck, a contractile sheath and a central tube has a length of approximately $130 \mathrm{~nm}$. Due to these morphological results and in accordance with the presence of dsDNA, the phage JG024 is grouped to the family Myoviridae. This family is a member of the order Caudovirales which contains exclusively tailed phages also from the families Siphoviridae and Podoviridae.

\section{Receptor of phage JG024}

We used different $P$. aeruginosa mutants to identify the receptor of phage JG024 as outlined by others [23]. Aflagella mutant $(\triangle f l i M)$, a pili mutant $(\triangle p i l A)$ and an LPS mutant $(\triangle a l g C)$ were infected with the phage JG024. After incubation, lysis was investigated on bacterial lawns (data not shown). JG024 lyses the pili- and the flagella mutant but not the $P$. aeruginosa $\triangle a l g C$ mutant. The algC gene encodes an enzyme with phosphoglucomutase and phosphomannomutase activity. A $P$. aeruginosa $\triangle a l g C$ mutant produces a truncated LPS core and lacks common antigen suggesting that these structures might constitute the host receptor for JG024 attachment $[24,25]$.

\section{Growth characteristics}

To investigate growth parameters like the latent phase and the burst size of the phage JG024, we performed

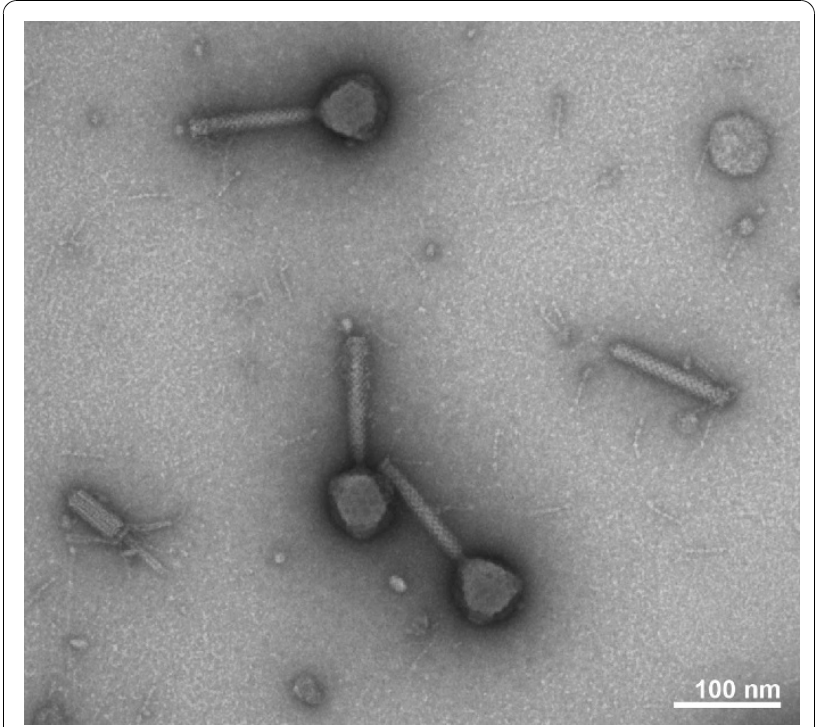

Figure 1 Morphology of JG024. Electron microscopic image of negatively stained JG024 phages, which exhibit a contractile tail with a length of $130 \mathrm{~nm}$. The icosahedral head of JG024 has a length of $80 \mathrm{~nm}$ and a width of $75 \mathrm{~nm}$. single step growth curves as described in Methods, Figure 2. Phage JG024 has an estimated latent phase of $50 \mathrm{~min}$. The burst size, which describes the mean number of phages liberated per bacterial cell was determined as 180 phages per infected cell.

\section{JG024 is a PB1-like phage}

Phage JG024 DNA was sequenced and assembled at McGill University as described in Methods. The genome size of phage JG024 is 66,275 bp and has a GC content of $55.62 \%$. Genome comparison using the blastx tool revealed that phage JG024 is highly related to the widespread and conserved PB1-like viruses $[15,26]$. It was recently reported that these phages show a high sequence similarity and limited horizontal gene transfer [15]. The general characteristics as well as the similarity to phage JG024 are shown in Table 2. The overall nucleotide similarity to PB1-like phages varies between $86 \%$ to phage PB1 and 95\% to the phages SN and 14-1 (Table 2). We also compared the JG024 genome sequence with PB1 and SN using Mauve [27] and detected only few insertions or deletions, Additional file 1 Figure S1. Due to the high sequence similarity, the broad host range characteristic as well as the morphology, we conclude that phage JG024 belongs to the PB1-like phages. In accordance with our findings, PB1-like phages also have been shown to use LPS as receptor [28]. Since the sampling location of JG024 in Lower Saxony, Germany is different to all other PB1-like phages, it underscores the broad environmental distribution of this phage group probably due to the broad host range [15].

\section{Features of the JG024 genome}

The schematic representation of the genome, with its assumed ORFs, some functional assignments and overall genetic organization is depicted in Figure 3. The genome of JG024 is compact organized with only $7.1 \%$

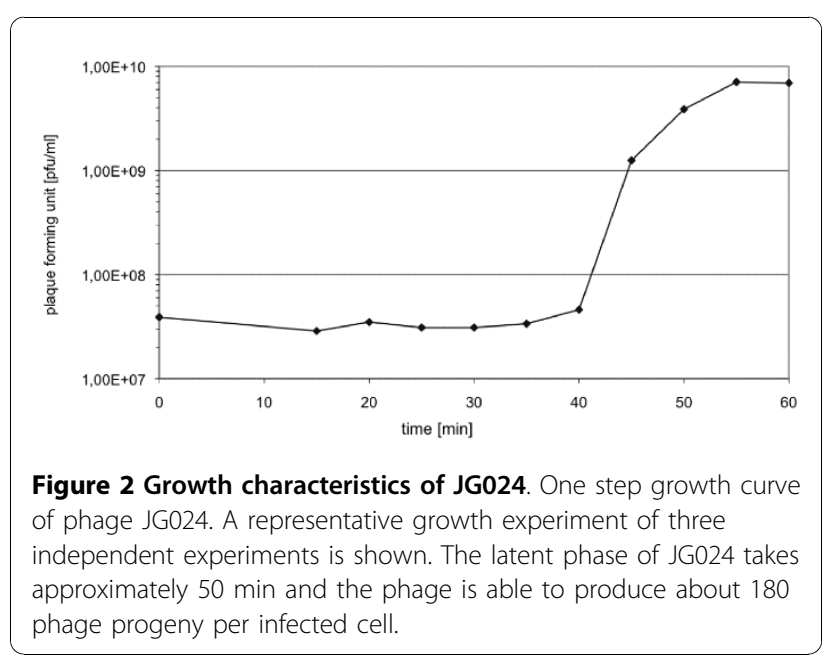


Table 2 Comparison of the JG024 genome to the genomes of PB1-like phages 15.

\begin{tabular}{cccccc}
\hline Phage & Genome size (bp) & GC content (\%) & Predicted ORFs & unique ORFs & DNA identity (\%) to JG024 \\
\hline JG024 & 66,275 & 55.62 & 94 & 1 & 100 \\
\hline PB1 & 65,764 & 55.5 & 93 & - & 86 \\
\hline F8 & 66,015 & 55.6 & 93 & 1 & 87 \\
\hline SN & 66,390 & 55.6 & 92 & 2 & 95 \\
\hline $14-1$ & 66,238 & 55.6 & 90 & - & 95 \\
\hline LMA2 & 66,530 & 55.5 & 95 & 2 & 93 \\
\hline LBL3 & 64,427 & 55.5 & 88 & 2 & 92 \\
\hline
\end{tabular}

intergenic space. No genes encoding for tRNAs were found in the genome of JG024 using the program RNAscan-SE 1.21 [29]. Interestingly, the GC content of phage JG024 differs from its host (55.62\% to 68\%). Comparison of the codon usage of JG024 with its host $P$. aeruginosa showed that the phage shares the same dominant codons for each amino acid except for valin, serin and glutamate. To test if the genome of phage JG024 is linear or circular, we used a method described previously [30]. A linear genome of phage JG024 was identified by treatment with exonuclease Bal31 which degrades only double-stranded linear DNA from both ends simultaneously (data not shown). However, we did not identify the exact genome ends. This would indicate that the genome of phage JG024 is circular permuted in contradiction to the PB1 phages, which have been reported to have non-permuted linear genomes [15]. Since the terminase protein of JG024 is highly (up to 99.6\%) identical to that of the PB1 phages, we assume phage JG024 to have a non-permuted linear genome.

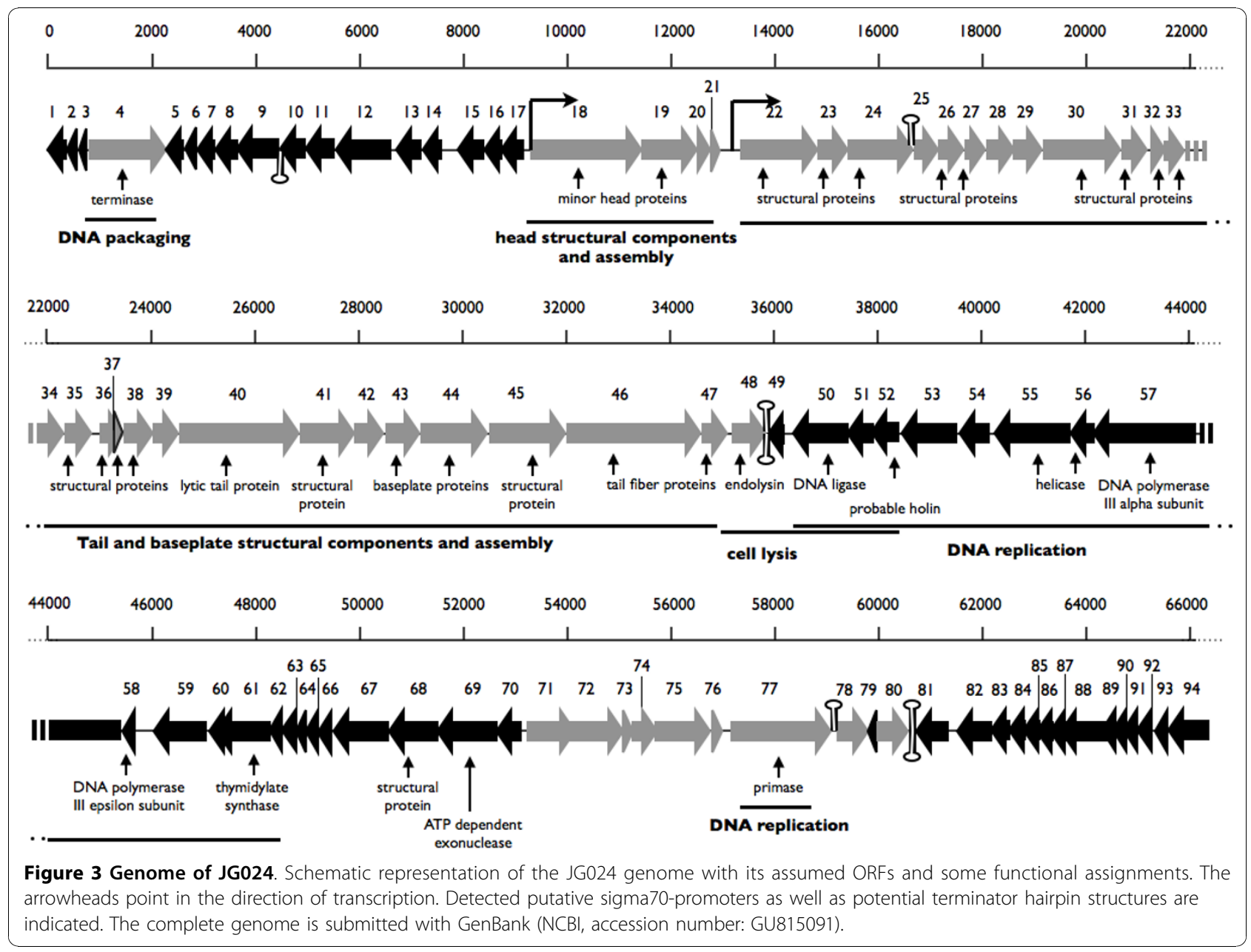


Since these phages share a high sequence similarity a comparative ORF prediction was possible. First, the heuristic approach of GeneMark was used to identify genes in small genomes under $100 \mathrm{~kb}$ [31]. With this approach a total of 84 putative ORFs were identified. In a second approach we used the NCBI ORF Finder program coupled with the program blastp and compared the translated proteins with the proteins of the PB1-like phages [26,32]. Combination of the results of both approaches revealed a total of 94 predicted ORFs as well as one unique ORF in phage JG024.

No RNA polymerase was detected suggesting that this phage uses the host transcriptional machinery, as it was also suggested for the PB1-like family of phages.

We detected a putative structural gene cluster which contains genes encoding for putative head structure proteins (ORF 18 and 19) as well as for tail and baseplate proteins (ORF 22-47). Moreover, ORF 40 was designated as a lytic tail protein. It was shown for the phages 14-1 and LBL3 that this protein has a transglycosylase domain with a $\mathrm{N}$-acetyl-D-glucosamine binding site, which shows a specific degradation of peptidoglycan [15]. ORF 48 encodes a putative endolysin with a high similarity to the endolysin of phage LMA2 (98.6\%) and belongs to a lysozyme-like superfamily. A putative holin may be encoded by ORF 52 , which shares a $100 \%$ identity to ORF 50 of phage F8 and to ORF 51 of phage 141. It was suggested that these ORFs encode probable holins since they are located near the endolysin gene and they encode a small protein (201 aa) containing three transmembrane domains [15].

Additionally, a complete DNA replication machinery was detected suggesting that the DNA replication is host independent as described for the PB1-like phages. The respective gene cluster contains a DNA ligase (ORF
50), a helicase (ORF 55 and 56), a DNA polymerase III (ORF 57 and 58), as well as a thymidylate synthase (ORF 61). A putative primase was also found but is not included in this gene cluster (ORF 77), as shown for the other PB1-like phages [15]. Also, differences between the PB1-like phages and JG024 were found. Phage 14-1 (ORF 71) and phage LBL3 (ORF 68) encode a hypothetical protein with a size of 434 aa. Interestingly, this protein is encoded by two ORFs in phage JG024 designated ORF 72 (362 aa) and 73 (60 aa). The two ORFs are separated by only $116 \mathrm{bp}$. Moreover, ORF 79 is a small predicted gene with a size of $132 \mathrm{bp}$ and encodes for a unique protein in phage JG024. This ORF was identified by two programs, GeneMark and ORF Finder, independently. No functional indication could be pointed out since there are no similarities to other proteins in the databases and no conserved domains have been detected in ORF 79. We also searched the genome of phage JG024 for promoters, terminators and regulatory elements, see Methods. The PB1 phages do not contain a phage RNA-polymerase and depend on the transcriptional machinery of the host bacterium. Putative sigma 70-promoter regions have been predicted in PB1 phages [15]. We detected two putative sigma 70-promoter regions in the 5'-region of ORF 18 and ORF 22, similar to the position of the putative promoters found in PB1 phages, see Table 3 and Figure 3[15]. In addition, we also determined the location of six rho-independent transcriptional terminators and checked if their position is conserved to the other PB1 like phages, Table 3 and Figure 3. Moreover, we searched for additional conserved motifs in intergenic regions using MEME and detected AT-rich boxes and additional conserved motifs in intergenic regions. However, the function of the motifs is unclear, their position indicates a possible

Table 3 Potential regulatory elements and intergenic motifs of the JG024 genome.

\begin{tabular}{|c|c|c|c|c|c|}
\hline Position & ORF & Sequence & Orientation & Score & $\mathrm{dG}\left(\mathrm{kcal} \mathrm{mol}^{-1}\right)$ \\
\hline \multicolumn{6}{|c|}{ putative $\sigma 70$-dependent promoter elements: } \\
\hline $9286 . .9336$ & ORF18 & ATGTTTGAATCTCITTTGAACGT TTGATGTTTCCCCTATAATAAGC GCACA & Forward & 1.22 & \\
\hline $13050 . .13100$ & ORF22 & TCATCTATAAGTAACGTTATAAC ATAACGTCAATTTATATGCTCTA GACGT & forward & 1.19 & \\
\hline \multicolumn{6}{|c|}{ putative rho-independent terminator elements: } \\
\hline $2313 . .2343$ & ORF 10 & AAGCCCGGAGCGATCCGGGCTTT TCTGTGTT & reverse & & -17.5 \\
\hline $16623 . .16644$ & ORF24 & GGCCGGGTTTCCGGCCTTTGTT & forward & & -12.3 \\
\hline $35910 . .35942$ & ORF48 & AAAAGGCCGCTTATTCAGCGGCC TTTTTGCTTT & forward & & -18.3 \\
\hline $35931 . .35900$ & ORF49 & AAAAGGCCGCTGAATAAGCGGCC TTTTCTTTT & reverse & & -18.3 \\
\hline $59033 . .59059$ & ORF77 & AGGCCGCCTTCGGGCGGTCTITT CTTT & forward & & -14.7 \\
\hline $60667 . .60706$ & ORF80 & AAAGCCCCGGACTCTAGTTCAGA ATCCGGGGCTTTCTTTT & forward & & -23.8 \\
\hline $60700 . .60657$ & ORF81 & AAAGCCCCGGATTCTGAACTAGA GTCCGGGGCTTTGTCGCTTCT & reverse & & -23.8 \\
\hline
\end{tabular}

Position and orientation of putative sigma 70 promoters and putative rho-independent terminator regions. The putative promoters were identified using SAK and Virtual Footprint as described in Methods. "Orf" indicates the Orf in the 3'-region of the putative promoter. Bold letters of the promoter sequences indicate -35 and -10 regions. The putative terminator regions were identified using the programs TransTerm and FindTerm as described in Methods. The indicated Orf is the respective Orf in the $5^{\prime}$-region of the putative rho-independent terminator. 
function as a recognition sequence for a phage sigma factor as suggested earlier [15].

\section{ASM infection assay}

Since phage JG024 is able to infect $84 \%$ of the tested clinical isolates in vitro we were interested if this phage is able to infect $P$. aeruginosa under simulated CF lung conditions. An artificial sputum medium (ASM) was used to mimic the CF lung environment. Growth in ASM leads to formation of typical biofilm-like microcolonies of $P$. aeruginosa and supports other phenotypic changes observed under chronic infection conditions [12]. At first, we tested the ability of phage JG024 to lyse the non-mucoid wild type strain $P$. aeruginosa PAO1 in ASM compared to LB medium. As described in Methods, we monitored phage particles and noted an increase of phage particles by a factor of nearly 500000 in LB and in ASM by a factor of 10000 (Figure 4). This indicates cell lysis by phage JG024 under these non ideal conditions (note that PAO1 was in stationary phase prior to infection to allow comparison to ASM). When we monitored infection of $P$. aeruginosa PAO1 in ASM we noticed a 50 -fold lower concentration of phage particles. This indicates a reduced efficiency of phage infection by JG024 under simulated chronic infection using the artificial sputum medium. In parallel we tested a $P$. aeruginosa CF-isolate, strain BT73, for susceptibility to phage infection in LB and ASM. Unexpectedly, we noticed only a 1.9 -fold lower phage number in ASM compared to LB (Figure 4). We noticed that phage JG024 was less effective against the CF isolate under both conditions, since approximately tenfold less phage particles were produced under both conditions

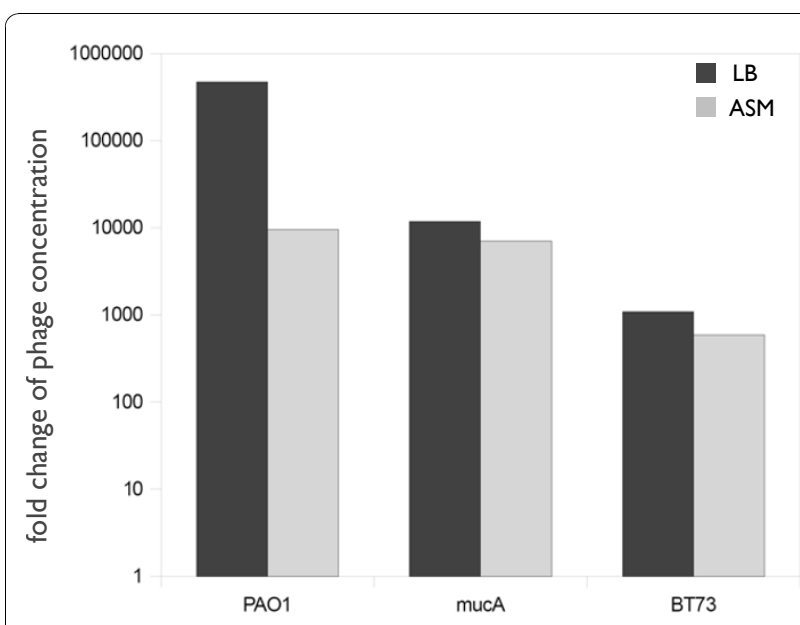

Figure 4 Infection assay of JG024 in ASM medium. Phage growth during infection assay in LB medium (dark grey bars) and ASM medium (light grey bars). Changes in phage concentration are described as $\mathrm{n}$-fold. compared to PAO1. However, while strain BT73 is less susceptible to phage lysis, the efficiency does not decrease dramatically under ASM growth conditions.

In contrast to the $P$. aeruginosa PAO1 strain the CFisolate BT73 is mucoid and secretes the exopolysaccharide alginate. We wondered if alginate overproduction could explain the observed results. It was recently published that even non-mucoid strains like the wild type PAO1 express the exopolysaccharide alginate in response to oxygen-limiting conditions [33]. We also observed that cultures of PAO1 in ASM, which mimics the CF lung, were highly viscous compared to the cultures in LB medium, suggesting a high production of alginate by the wild type PAO1 in this medium. If alginate is the factor in our experimental setup which decreases phage infection efficiency, a mucoid variant of strain PAO1 should show a similar result as the clinical isolate BT73. Therefore, we repeated the phage infection experiments in LB and ASM with a $P$. aeruginosa mucA mutant strain. We observed again only a 1.6 -fold decrease in ASM and an overall approximately tenfold reduction in phage particles when compared with P. aeruginosa PAO1 (Figure 4). These results are in agreement with our hypothesis that alginate overproduction reduces phage infection efficiency. Moreover, they point to alginate as the dominant factor for the decrease in phage infection efficiency in ASM. To verify this result, we performed the same experiment with $P$. aeruginosa PAO1 in LB medium and increasing alginate concentrations. We chose alginate concentrations of 50 , $100,200,500 \mu \mathrm{g} / \mathrm{ml}$ up to $1000 \mu \mathrm{g} / \mathrm{ml}$, since nonmucoid $P$. aeruginosa strains have been reported to produce $50-200 \mu \mathrm{g} / \mathrm{ml}$ alginate, while mucoid isolates produce up to $1000 \mu \mathrm{g} / \mathrm{ml}$ alginate [34-36]. In accordance with our hypothesis, the presence of alginate reduced phage multiplication in our test assay. A concentration of 50 to $200 \mu \mathrm{g} / \mathrm{ml}$ alginate resulted in an almost 20fold reduction of phage particles compared to LB medium alone in accordance with the 50-fold reduction of phage particles observed in ASM compared to LB. This effect is even more obvious with alginate concentations of 500 and $1000 \mu \mathrm{g} / \mathrm{ml}$, where we observed a reduction of phage particles by a factor of 130 and almost 2800, respectively.

\section{Conclusions}

We could show that the phage JG024 belongs to the PB1-like phages and shares several characteristic features of this group. These phages are widespread in nature and very successful. A new member of this group, phage JG024, was isolated and characterized. General growth characteristics as well as the genome were investigated, showing that JG024 is able to pass one infection cycle in approximately $50 \mathrm{~min}$. Genome analysis 
revealed the strong relatedness to the PB1-like phages. Moreover, we could show that JG024 has broad spectrum activity with a prevalence to clinical isolates. Also, infection of the host $P$. aeruginosa was even possible under challenging conditions like the ASM medium which mimics the CF lung. High viscosity and microcolony growth of the host were only small obstacles for JG024 to infect and multiply under these conditions. These results show that this group of bacteria could be an important contribution to phage therapy. Moreover, we established a method to investigate the possibility of a phage to lyse bacteria under infection conditions prior to use for phage therapy in vivo.

\section{Methods}

\section{Bacterial strains and growth conditions}

Table 1 shows the genotype and phenotypes of the bacteria and phage JG024 used in this study. The 100 environmental Pseudomonas aeruginosa strains used in this study origin from a comprehensive screen of approx. 400 environmental river strains. These were genetically characterized using the ArrayTube hybridization chip [37]. The 100 strains used here are all different in their core genomic SNP pattern and were chosen such to represent the entire population genetic diversity currently known for $P$. aeruginosa. Details of the comprehensive screen will be published elsewhere. $P$. aeruginosa strains were routinely propagated in Luria Bertani (LB) broth medium aerobically at $37^{\circ} \mathrm{C}$. The composition of the artificial sputum medium (ASM) is described elsewhere [12].

\section{Phage Isolation}

Phages were isolated from sewage following a simple enrichment procedure. Samples from a sewage plant Steinhof in Braunschweig, Germany were centrifuged for $5 \mathrm{~min}$ at $4100 \times \mathrm{g}$ (Biofuge fresco). Ten $\mathrm{ml}$ of the supernatant were mixed with $5 \mathrm{ml}$ of a $P$. aeruginosa overnight culture and incubated in $50 \mathrm{ml} \mathrm{LB}$ broth at room temperature. After an incubation of $48 \mathrm{~h}$, the cells were sedimented by centrifugation at $4100 \times \mathrm{g}$ (Biofuge fresco) for $10 \mathrm{~min}$ and the supernatant was transferred to a clean tube. To kill remaining bacteria, several drops of chloroform were added to the supernatant and the emulsion was mixed for $30 \mathrm{~s}$. To separate the phages, appropriate dilutions of the phage lysate were spotted onto bacterial lawns of top-agar plates. Top-agar plates were produced by adding approximately $5^{*} 10^{8}$ cells $/ \mathrm{ml}$ of $P$. aeruginosa from an overnight LB broth to $3.5 \mathrm{ml}$ of LB top-agar $(0.75 \%)$. The inoculated top-agar was overlaid on an LB agar plate and allowed to solidify. After incubation at $37^{\circ} \mathrm{C}$ for 10 to $16 \mathrm{~h}$ zones of lysis were monitored. Single plaques, derived from a single phage, were separated by stinging with a pipette tip into the plaque followed by resuspending the phages in SM buffer $(100 \mathrm{mM} \mathrm{NaCl}, 8 \mathrm{mM} \mathrm{MgSO}$, $50 \mathrm{mM}$ Tris- $\mathrm{HCl}$, $\mathrm{pH}$ 7.5). The resulting phage lysate was stored at $4^{\circ} \mathrm{C}$.

\section{Electron microscopy}

The morphology of the phages was detected by negative staining with uranyl acetate and transmission electron microscopy. Phages were allowed to absorbe onto a thin carbon film, prepared on mica, from a liquid sample for different time points, washed in TE buffer $(10 \mathrm{mM}$ TRIS, 2 mM EDTA, pH 6.9) and distilled water. Phages were negatively stained by floating the carbon film for approx. $15 \mathrm{sec}$ on a drop of $2 \%$ aqueous uranyl acetate. Then, the carbon film was picked up with copper grids (300 mesh), blotted semi-dry with filter paper and was subsequently air dried. Samples were examined in a Zeiss EM910 transmission electron microsope at an acceleration voltage of $80 \mathrm{kV}$ and at calibrated magnifications. Images were recorded digitally with a Slow-Scan CCD-Camera (ProScan, $1024 \times 1024$, Scheuring, Germany) with ITEM-Software (Olympus Soft Imaging Solutions, Münster, Germany). Brightness and contrast were adjusted with Adobe Photoshop CS3.

\section{Phage host range spectrum and detection of host receptor}

To determine the phage host range, top-agar plates with the potential host lawn were prepared. Top-agar plates were produced by adding approximately $5 * 10^{8}$ cells $/ \mathrm{ml}$ of $P$. aeruginosa from an overnight LB broth to $3.5 \mathrm{ml}$ of LB top agar (0.75\%). Ten $\mu \mathrm{l}$ of a phage stock solution were spotted on the top-agar plate and incubated at $37^{\circ} \mathrm{C}$ for 12 to $16 \mathrm{~h}$. After incubation, the appearance of the lysis zones at the site where the phage suspension was added, was examined. Each phage was tested against each bacterial strain in triplicate in independent experiments. The lysis was categorized as clear $(+)$, turbid $(0)$ and no reaction (-) as described [38]. For detection of the phage receptor molecule, we used a $P$. aeruginosa flagella mutant $(\triangle f l i M)$, a pili mutant $(\triangle p i l A)$ and an LPS mutant $(\triangle a l g C)$, which were infected with the phage JG024 as described above. The strains for the receptor identification are derived from a PAO1 wildtype and therefore belong to the same serotype as PAO1, namely serotype O5 [39]. An effect on the efficiency of plating was not observed for the strains with intact LPS.

\section{Phage growth characteristics}

To determine phage growth characteristics like burst size and duration of the infection cycle, single step growth experiments were performed as previously described with some modifications $[40,41]$. P. aeruginosa was grown aerobically in $10 \mathrm{ml} \mathrm{LB}$ medium until 
exponential growth phase. After the bacteria reached an OD578 of 0.3 , an aliquot containing $5^{*} 10^{8}$ phages was added to the culture which corresponds to a multiplicity of infection (MOI) of 0.16. Therefore, it is likely that a cell is infected by only one phage and that the amount of infected bacteria is equal to the amount of the initial phage concentration. After addition of the phages, one aliquot was immediately used for determination of the phage titer. Then, phages were allowed to adsorb for 15 min. Afterwards, cultures were diluted in LB $10^{4}-, 10^{5}$-, $10^{6}$ - and $10^{7}$-fold and incubated at $37^{\circ} \mathrm{C}$ for $60 \mathrm{~min}$. Samples for phage enumeration were taken aseptically at different time points after infection. The burst size was determined as: (phage titer at the end of the single step growth curve at time point 55 min minus phage titer at time point $20 \mathrm{~min}$ ) divided by phage titer at time point $20 \mathrm{~min}$. The latent phase was estimated at the midpoint of the exponential phase of a one step growth experiment [40,41].

\section{Sequencing, analysis and annotation of phage genomes}

To isolate phage DNA, phages were propagated in topagar plates as described above. After growth at $37^{\circ} \mathrm{C}$ the plates were overlayed with $10 \mathrm{ml}$ SM buffer and incubated with shaking at $4^{\circ} \mathrm{C}$ for $4 \mathrm{~h}$. The supernatant was sterile filtrated $(0.22 \mu \mathrm{m})$ and stored at $4^{\circ} \mathrm{C}$. Phage DNA was isolated using the Qiagen Lambda Kit according to manufacturer's instructions. Ten $\mathrm{ml}$ phage lysate with a titer of at least $1^{*} 10^{10}$ phages $/ \mathrm{ml}$ were used to isolate up to $1 \mu \mathrm{g} / \mu \mathrm{l}$ pure phage DNA. Digestion with restriction endonucleases was done following the protocols of the manufacturer. Whole genome sequencing of the phage JG024 was done at the McGill University and Génome Québec Innovation Centre (Montréal, QC, Canada) using the Genome Sequencer FLX and 454 Technology. A total of 66,684 reads with an average length of 344 bases was assembled to one single contig with a 300 fold coverage. The annotation of the unknown phage genes was done by using the software GeneMark.HMM [31]. The Heuristic approach of GeneMark was used to identify genes in small genomes under $100 \mathrm{~kb}$. The identified genes were compared with the NCBI ORF Finder [32]. Nucleotide sequences were scanned for homologues using the Basic Alignment Search Tool (blastx) [26]. To search for tRNA genes in the phage sequences the internet tool tRNAscan-SE 1.21 was used [29]. Sequence comparison was conducted using ClustalW2 online analysis tool [42]. Investigation of the codon usage was performed using a software tool based on JCat [43]. The genome sequence as well as the annotation is deposited with the GenBank (National Center for Biotechnology Information) using the following accession number: GU815091.

\section{Identification of promoter regions, terminator structures} and other motifs

The genome of phage JG024 was scanned for the presence of sigma 70-dependent promoter regions using the web service SAK [44]. Putative promoter regions with a score above 1 were scanned for the presence of conserved -10 and -35 regions using the Virtual Footprint software [45]. Two promoter regions were identified in this way. Rho-independent terminator structures were identified using the TransTerm [46] and FindTerm (Softberry, Inc.) software tools. The program MEME was used for identification of conserved intergenic motifs in phage JG024 [47].

\section{ASM infection assay}

Phage susceptibility of $P$. aeruginosa in ASM medium was tested in 24 well plates. $1 \mathrm{ml}$ ASM medium and as control LB medium were inoculated with indicated strains aerobically for $24 \mathrm{~h}$ at $37^{\circ} \mathrm{C}$. An OD 578 of 0.5 was used for the inoculation. Afterwards, $1^{*} 10^{5}$ phages were added which describes the initial phage concentration. After incubation for additional $24 \mathrm{~h}$ at $37^{\circ} \mathrm{C}$ the colony forming units (CFU) as well as the plaque forming units (PFU) were determined. To determine the change in phage concentration we divided the final phage concentration after $24 \mathrm{~h}$ by the initial phage concentration. To determine the effect of alginate the same experiment was performed in LB with purified alginate using increasing concentrations in a range of $50 \mu \mathrm{g} / \mathrm{ml}$ to $1 \mathrm{mg} / \mathrm{ml}$. Alginate was purified from mucoid P. aeruginosa strain FRD1 [34] as described previously [36].

\section{Additional material}

Additional file 1: Supplementary Figure S1. Graph and schematic representation of a Mauve comparison using phage JG024, phage PB1 and SN.

\section{Acknowledgements}

The authors thank Gerd Döring, Burkhard Tümmler and Michael Hogardt for providing the clinical $P$. aeruginosa strains. We thank Petra Tielen for the gift of isolated alginate. JG was supported by the DFG-European Graduate College 653.

\section{Author details}

${ }^{1}$ Institute of Microbiology, Technische Universität Braunschweig, Spielmannstr. 7, 38106 Braunschweig, Germany. ${ }^{2}$ DSMZ, German Collection of Microorganisms and Cell Cultures, Inhoffenstr. 7B, 38124 Braunschweig, Germany. ${ }^{3} \mathrm{HZI}$, Helmholtz Centre for Infection Research, Inhoffenstr. 7, 38124 Braunschweig, Germany.

\section{Authors' contributions}

$J G$ participated in the design of the study, isolated and characterized the phages, annotated the genome, performed host specificity observations of clinical isolates as well as the ASM assay and drafted the manuscript. AW provided the ASM medium and participated in the ASM assay. BB assisted with bioinformatic analyses. MK, KS, CR and JS were involved in the host 
specificity study of the 100 environmental strains which were provided and investigated by KS and JS. Electron microscopically examinations were done by MR. DJ contributed to the design of the study. MS designed the study, carried out bioinformatic analysis and revised the manuscript. All authors read and approved the final manuscript.

Received: 21 May 2010 Accepted: 26 November 2010 Published: 26 November 2010

\section{References}

1. Schweizer HP: Efflux as a mechanism of resistance to antimicrobials in Pseudomonas aeruginosa and related bacteria: unanswered questions. Genet Mol Res 2003, 2:48-62.

2. Lyczak JB, Cannon CL, Pier GB: Lung infections associated with cystic fibrosis. Clin Microbiol Rev 2002, 15:194-222.

3. Puzová H, Siegfried L, Kmetová M, Durovicová J, Kerestesová A: Characteristics of Pseudomonas aeruginosa strains isolated from urinary tract infections. Folia Microbiol (Praha) 1994, 39:337-341.

4. Sadikot RT, Blackwell TS, Christman JW, Prince AS: Pathogen-host interactions in Pseudomonas aeruginosa pneumonia. Am J Respir Crit Care Med 2005, 171:1209-1223.

5. Church D, Elsayed S, Reid O, Winston B, Lindsay R: Burn wound infections. Clin Microbiol Rev 2006, 19:403-434.

6. Campodónico VL, Gadjeva M, Paradis-Bleau C, Uluer A, Pier GB: Airway epithelial control of Pseudomonas aeruginosa infection in cystic fibrosis. Trends Mol Med 2008, 14:120-133.

7. Döring G, Gulbins E: Cystic fibrosis and innate immunity: how chloride channel mutations provoke lung disease. Cell Microbiol 2009, 11:208-216.

8. Riordan JR, Rommens JM, Kerem B, Alon N, Rozmahel R, Grzelczak Z, Zielenski J, Lok S, Plavsic N, Chou JL: Identification of the cystic fibrosis gene: cloning and characterization of complementary DNA. Science 1989, 245:1066-1073.

9. Singh PK, Schaefer AL, Parsek MR, Moninger TO, Welsh MJ, Greenberg EP: Quorum-sensing signals indicate that cystic brosis lungs are infected with bacterial biofilms. Nature 2000, 407:762-764.

10. Mah TF, O'Toole GA: Mechanisms of biofilm resistance to antimicrobial agents. Trends Microbiol 2001, 9:34-39.

11. Palmer KL, Mashburn LM, Singh PK, Whiteley M: Cystic fibrosis sputum supports growth and cues key aspects of Pseudomonas aeruginosa physiology. J Bacteriol 2005, 187:5267-5277.

12. Sriramulu DD, Lünsdorf $H$, Lam JS, Römling U: Microcolony formation: a novel biofilm model of Pseudomonas aeruginosa for the cystic brosis lung. J Med Microbiol 2005, 54:667-676.

13. Matsui $H$, Wagner VE, Hill DB, Schwab UE, Rogers TD, Button B, Taylor RM Superfine R, Rubinstein M, Iglewski BH, Boucher RC: A physical linkage between cystic fibrosis airway surface dehydration and Pseudomonas aeruginosa biofilms. Proc Natl Acad Sci USA 2006, 103:18131-18136.

14. Sulakvelidze A, Alavidze Z, Morris JG: Bacteriophage therapy. Antimicrob Agents Chemother 2001, 45:649-659.

15. Ceyssens P, Miroshnikov K, Mattheus W, Krylov V, Robben J, Noben J, Vanderschraeghe S, Sykilinda N, Kropinski A, Volckaert G, Mesyanzhinov V, Lavigne R: Comparative analysis of the widespread and conserved PB1like viruses infecting Pseudomonas aeruginosa. Environ Microbiol 2009, 11:2874-2883.

16. Krylov VN, Tolmachova TO, Akhverdian VZ: DNA homology in species of bacteriophages active on Pseudomonas aeruginosa. Arch Virol 1993, 131:141-151

17. Merabishvili M, Pirnay JP, Verbeken $G$, Chanishvili N, Tediashvili M, Lashkhi N, Glonti T, Krylov V, Mast J, Parys LV, Lavigne R, Volckaert G, Mattheus W, Verween G, Corte PD, Rose T, Jennes S, Zizi M, Vos DD, Vaneechoutte M: Quality-controlled small-scale production of a welldefined bacteriophage cocktail for use in human clinical trials. PLOS ONE 2009, 4:e4944.

18. Skurnik M, Strauch E: Phage therapy: facts and fiction. Int J Med Microbiol 2006, 296:5-14.

19. Levin BR, Bull JJ: Population and evolutionary dynamics of phage therapy. Nat Rev Micro 2004, 2:166-173.

20. Martin DW, Schurr MJ, Mudd MH, Govan JR, Holloway BW, Deretic V: Mechanism of conversion to mucoidy in Pseudomonas aeruginosa infecting cystic fibrosis patients. Proc Natl Acad Sci USA 1993, 90:8377-8381.
21. Hassett DJ, Sutton MD, Schurr MJ, Herr AB, Caldwell CC, Matu JO: Pseudomonas aeruginosa hypoxic or anaerobic biofilm infections within cystic fibrosis airways. Trends Microbiol 2009, 17:130-138.

22. Ackermann HW: 5500 Phages examined in the electron microscope. Arch Virol 2007, 152:227-243.

23. Budzik JM, Rosche WA, Rietsch A, OToole GA: Isolation and characterization of a generalized transducing phage for Pseudomonas aeruginosa strains PAO1 and PA14. J Bacteriol 2004, 186:3270-3273.

24. Coyne MJ, Russell KS, Coyle CL, Goldberg JB: The Pseudomonas aeruginosa $\mathrm{alg} \mathrm{C}$ gene encodes phosphoglucomutase, required for the synthesis of a complete lipopolysaccharide core. J Bacterio/ 1994, 176:3500-3507.

25. King J, Kocíncová D, Westman E, Lam J: Lipopolysaccharide biosynthesis in Pseudomonas aeruginosa. Innate Immun 2009, 15:261-312.

26. Altschul SF, Madden TL, Schäffer AA, Zhang J, Zhang Z, Miller W, Lipman DJ: Gapped BLAST and PSI-BLAST: a new generation of protein database search programs. Nucleic Acids Res 1997, 25:3389-3402.

27. Darling ACE, Mau B, Blattner FR, Perna NT: Mauve: multiple alignment of conserved genomic sequence with rearrangements. Genome Res 2004, 14:1394-1403.

28. Jarrell K, Kropinski AM: Identification of the cell wall receptor for bacteriophage E79 in Pseudomonas aeruginosa strain PAO. J Virol 1977, 23:461-466.

29. Lowe TM, Eddy SR: tRNAscan-SE: a program for improved detection of transfer RNA genes in genomic sequence. Nucleic Acids Res 1997, 25:955-964.

30. Loessner MJ, Inman RB, Lauer P, Calendar R: Complete nucleotide sequence, molecular analysis and genome structure of bacteriophage A118 of Listeria monocytogenes: implications for phage evolution. Mol Microbiol 2000, 35:324-340.

31. Besemer J, Borodovsky M: Heuristic approach to deriving models for gene finding. Nucleic Acids Res 1999, 27:3911-3920.

32. Wheeler DL, Church DM, Federhen S, Lash AE, Madden TL, Pontius JU, Schuler GD, Schriml LM, Sequeira E, Tatusova TA, Wagner L: Database resources of the National Center for Biotechnology. Nucleic Acids Res 2003, 31:28-33.

33. Bragonzi A, Worlitzsch $D$, Pier GB, Timpert $P$, Ulrich $M$, Hentzer $M$, Andersen JB, Givskov M, Conese M, Doring G: Nonmucoid Pseudomonas aeruginosa expresses alginate in the lungs of patients with cystic fibrosis and in a mouse model. J Infect Dis 2005, 192:410-419.

34. Ohman DE, Chakrabarty AM: Genetic mapping of chromosomal determinants for the production of the exopolysaccharide alginate in a Pseudomonas aeruginosa cystic fibrosis isolate. Infect Immun 1981, 33:142-148.

35. Tielen $\mathrm{P}$, Rosenau $\mathrm{F}$, Wilhelm $\mathrm{S}$, Jaeger KE, Flemming HC, Wingender J: Extracellular enzymes affect biofilm formation of mucoid Pseudomonas aeruginosa. Microbiology 2010, 156:2239-2252.

36. Wingender J, Strathmann M, Rode A, Leis A, Flemming HC: Isolation and biochemical characterization of extracellular polymeric substances from Pseudomonas aeruginosa. Meth Enzymol 2001, 336:302-314.

37. Wiehlmann L, Wagner G, Cramer N, Siebert B, Gudowius P, Morales G, Kohler $T$, van Delden C, Weinel C, Slickers $P$, Tummler B: Population structure of Pseudomonas aeruginosa. Proc Natl Acad Sci USA 2007, 104:8101-8106.

38. Knezevic P, Kostanisek R, Obreht D, Petrovic O: Isolation of Pseudomonas aeruginosa specific phages with broad activity spectra. Curr Microbiol 2009, 59:173-180.

39. de Kievit TR, Dasgupta T, Schweizer H, Lam JS: Molecular cloning and characterization of the rfc gene of Pseudomonas aeruginosa (serotype O5). Mol Microbiol 1995, 16:565-574.

40. Pajunen M, Kiljunen S, Skurnik M: Bacteriophage phiYeO3-12, specific for Yersinia enterocolitica serotype 0:3, is related to coliphages T3 and T7. J Bacteriol 2000, 182:5114-5120.

41. Moineau S, Durmaz E, Pandian S, Klaenhammer TR: Differentiation of Two Abortive Mechanisms by Using Monoclonal Antibodies Directed toward Lactococcal Bacteriophage Capsid Proteins. Appl Environ Microbiol 1993, 59:208-212.

42. Larkin MA, Blackshields G, Brown NP, Chenna R, McGettigan PA, McWilliam H, Valentin F, Wallace IM, Wilm A, Lopez R, Thompson JD, Gibson TJ, Higgins DG: Clustal W and Clustal $\times$ version 2.0. Bioinformatics 2007, 23:2947-2948. 
43. Grote A, Hiller K, Scheer M, Munch R, Nörtemann B, Hempel DC, Jahn D: JCat: a novel tool to adapt codon usage of a target gene to its potential expression host. Nucleic Acids Res 2005, 33:W526-531.

44. Gordon L, Chervonenkis AY, Gammerman AJ, Shahmuradov IA, Solovyev W: Sequence alignment kernel for recognition of promoter regions. Bioinformatics 2003, 19:1964-1971.

45. Münch R, Hiller K, Grote A, Scheer M, Klein J, Schobert M, Jahn D: Virtual Footprint and PRODORIC: an integrative framework for regulon prediction in prokaryotes. Bioinformatics 2005, 21:4187-4189.

46. Ermolaeva MD, Khalak HG, White O, Smith HO, Salzberg SL: Prediction of transcription terminators in bacterial genomes. J Mol Biol 2000, 301:27-33.

47. Bailey $T L$, Elkan C: Fitting a mixture model by expectation maximization to discover motifs in biopolymers. Proc Int Conf Intell Syst Mol Biol 1994, 2:28-36.

48. Dunn NW, Holloway BW: Pleiotrophy of p-fluorophenylalanine-resistant and antibiotic hypersensitive mutants of Pseudomonas aeruginosa. Genet Res 1971, 18:185-197.

49. Rahme LG, Stevens EJ, Wolfort SF, Shao J, Tompkins RG, Ausubel FM: Common virulence factors for bacterial pathogenicity in plants and animals. Science 1995, 268:1899-1902.

50. Klausen M, Heydorn A, Ragas P, Lambertsen L, Aaes-Jørgensen A, Molin S, Tolker-Nielsen T: Biofilm formation by Pseudomonas aeruginosa wild type, agella and type IV pili mutants. Mol Microbiol 2003, 48:1511-1524.

doi:10.1186/1471-2180-10-301

Cite this article as: Garbe et al: Characterization of JG024, a

pseudomonas aeruginosa PB1-like broad host range phage under simulated infection conditions. BMC Microbiology 2010 10:301.

\section{Submit your next manuscript to BioMed Central} and take full advantage of:

- Convenient online submission

- Thorough peer review

- No space constraints or color figure charges

- Immediate publication on acceptance

- Inclusion in PubMed, CAS, Scopus and Google Scholar

- Research which is freely available for redistribution

Submit your manuscript at www.biomedcentral.com/submit
Ciomed Central 